\title{
THE FOURTH ORDER ACCURACY DECOMPOSITION SCHEME FOR AN EVOLUTION PROBLEM
}

\author{
Zurab Gegechkori $^{1}$, Jemal Rogava ${ }^{1}$ and Mikheil Tsiklauri ${ }^{1}$
}

\begin{abstract}
In the present work, the symmetrized sequential-parallel decomposition method with the fourth order accuracy for the solution of Cauchy abstract problem with an operator under a split form is presented. The fourth order accuracy is reached by introducing a complex coefficient with the positive real part. For the considered scheme, the explicit a priori estimate is obtained.
\end{abstract}

Mathematics Subject Classification. 65M12, 65M15, 65M55.

Received: July 31, 2003. Revised: May 25, 2004.

\section{INTRODUCTION}

It is known that mathematical simulation of processes taking place in the nature frequently leads to consideration of boundary-value problems for partial-differential evolution (nonstationary) equation. These kind of problems can be considered as a Cauchy abstract problem in a Banach space for an evolution equation with an unbounded operator.

Study of approximated schemes for solution of evolution problems leads to the conclusion that a certain operator (solution operator of the discrete problem) corresponds to each approximated scheme. This operator approximates the solution operator (semigroup) of the initial continuous problem. For example, if we use the Rotte scheme for the solution of an evolution problem, then the solution operator of the difference problem thus obtained will be a discrete semigroup and we will have the approximation of a continuous semigroup by discrete semigroups (see [32], Ch. IX). On the other hand, on the basis of the approximation of a continuous semigroup, we can construct an approximated scheme for solution of an evolution problem.

Decomposition formulas approximate a continuous semigroup by means of the combination of the semigroups generated by the addends of the operator generating this semigroup.

The first decomposition formula for an exponential matrix function was constructed by Lie in 1875 . Trotter generalized this formula for an exponential operator function-semigroup in 1959 [49]. The resolvent analogue of this formula for the first time was constructed by Chernoff in $1968[6,7]$. At the same time, in the sixties of the xxth century, in order to elaborate numerical methods for solution of multi-dimensional problems of mathematical physics, the subject of construction of decomposition schemes has naturally raised. Decomposition schemes allow to reduce a solution of multi-dimensional problems to the solution of one-dimensional problems.

Keywords and phrases. Decomposition method, semigroup, operator split method, Trotter formula, Cauchy abstract problem.

1 I. Vekua Institute of Applied Mathematics, University Str. 2, 0143, Tbilisi, Georgia.

e-mail: GegeZu.Cyber@viam.hepi.edu.ge, JRogava@viam.hepi.edu.ge, MTsikla@viam.hepi.edu.ge

(C) EDP Sciences, SMAI 2004 
First works dedicated to construction and investigation of decomposition schemes were published in the fifties and sixties of the xxth century (see Andreev [1], Baker [2], Baker and Oliphant [3], Birkhoff and Varga [4], Birkhoff, Varga and Young [5], Douglas [11], Douglas and Rachford [12], Diakonov [9,10], Dryja [13], Fairweather, Gourlay and Mitchell [14], Fryazinov [15], Gordeziani [20], Gourlay and Mitchell [24], Ianenko [25, 26], Ianenko and Demidov [27], Konovalov [33], Marchuk and Ianenko [37], Marchuk and Sultangazin [38], Peaceman and Rachford [39], Ilin [30], Samarskii [43-45], and Temam [48]). These works became a basis of the further investigation of decomposition schemes.

We can show that the split problem, obtained by means of a decomposition method, generates the Trotter formula (see Trotter [49]), or the Chernoff formula (see Chernoff $[6,7]$ ), or a formula which is a combination of these formulas. Thus, an estimate of decomposition formula's error is equivalent to the study of approximation of continuous semigroup by Lie-Trotter and Lie-Chernoff type formulas. The works of Ichinose and Takanobu [28], Ichinose and Tamura [29], Rogava [41], (see also [42], T. II) are devoted to estimate of error of Lie-Trotter and Lie-Chernoff type formulas.

We call Lie-Trotter type formulas the formulas which approximate a semigroup by a combination of semigroups generated by the addends of the operator generating this semigroup.

We call Lie-Chernoff type formulas the formulas which are obtained from Lie-Trotter type formulas if we replace semigroups by the corresponding resolvents.

Decomposition schemes conditionally can be divided into two groups: differential and difference. Lie-Trotter type formulas correspond to differential decomposition schemes and Lie-Chernoff type formulas to difference schemes.

Decomposition schemes, associated with the Lie and Trotter formulas, allow to split a Cauchy problem for an evolution equation with the operator $A=A_{1}+A_{2}$ into two problems, respectively with the operators $A_{1}$ and $A_{2}$, which are solved sequentially on the time interval with the length $t / n$.

Decomposition schemes associated with the Chernoff formula are known as the fractional-step method (see Ianenko [26]).

Decomposition schemes in view of numerical calculation can be divided into two groups: schemes of sequential account (see for example Marchuk [36]) and schemes of parallel account (Gordeziani and Meladze [22, 23], Gordeziani and Samarskii [21], Kuzyk and Makarov [35]). In [42] (see Sect. II), there are obtained explicit a priori estimates for decomposition schemes of parallel account considered in [22]. There exist a lot of works devoted to decomposition schemes. For example, see $[26,36,46]$ and the references therein.

The accuracy order of the above-mentioned schemes are first or second. As it is known to us, the high accuracy order decomposition schemes in case of two addends $\left(A=A_{1}+A_{2}\right)$ for the first time were obtained by Dia and Schatzman (see [8]). Note that the formulas constructed in these works are not automatically stable decomposition formulas. Decomposition formula is called automatically stable if a sum of the absolute values of split coefficients is equal to one. Sheng has proved [47] that, on the real number field, there does not exist such automatically stable splitting of $\exp (-t A)$, the accuracy order of which is higher than two.

The present work is devoted to construction and investigation of the high order accuracy decomposition scheme for an evolution problem.

In this work, by introducing a complex parameter, the fourth order accuracy symmetrized sequential-parallel type differential scheme is constructed for a two-dimensional evolution problem with a split operator. The main operator of the evolution problem conditionally is called the two-dimensional split operator if it represents a sum of two addends $\left(A=A_{1}+A_{2}\right)$. The formula, corresponding to the constructed scheme, is an automatically stable decomposition formula. For the considered scheme, there is obtained an explicit a priori estimate. Under the explicit estimate we mean such a priori estimate for the solution error, where constants in the righthand side do not depend on the solution of the initial continuous problem, i.e. are absolute constants. In the works [16-19], we have constructed the third order accuracy decomposition schemes for two- and multidimensional homogeneous and inhomogeneous evolution problems. 


\section{Statement of the Problem}

Let us consider the Cauchy problem for an evolution equation in the Banach space $X$ :

$$
\frac{\mathrm{d} u(t)}{\mathrm{d} t}+A u(t)=0, \quad t>0, \quad u(0)=\varphi
$$

where $A$ is a linear closed operator with a definition domain $D(A)$, which is everywhere dense in $X, \varphi$ is a given element from $D(A)$.

Suppose that the operator $(-A)$ generates a strongly continuous semigroup $\{\exp (-t A)\}_{t \geq 0}$. Then the solution of problem (1) is given by the following formula $[1,36,37]$ :

$$
u(t)=U(t, A) \varphi,
$$

where $U(t, A)=\exp (-t A)$ is a strongly continuous semigroup.

Let $A=A_{1}+A_{2}$, where $A_{i} \quad(i=1,2) \quad$ are closed operators, densely defined in $X$.

Let us introduce a grid set:

$$
\bar{\omega}_{\tau}=\left\{t_{k}=k \tau, k=1,2, \ldots, \tau>0\right\} .
$$

Together with problem (1), on each interval $\left[t_{k-1}, t_{k}\right]$, we consider a sequence of the following problems:

$$
\begin{aligned}
& \frac{\mathrm{d} v_{k}^{(1)}(t)}{\mathrm{d} t}+\frac{\alpha}{2} A_{1} v_{k}^{(1)}(t)=0, \quad v_{k}^{(1)}\left(t_{k-1}\right)=u_{k-1}\left(t_{k-1}\right), \\
& \frac{\mathrm{d} v_{k}^{(2)}(t)}{\mathrm{d} t}+\frac{1}{2} A_{2} v_{k}^{(2)}(t)=0, \quad v_{k}^{(2)}\left(t_{k-1}\right)=v_{k}^{(1)}\left(t_{k}\right), \\
& \frac{\mathrm{d} v_{k}^{(3)}(t)}{\mathrm{d} t}+\bar{\alpha} A_{1} v_{k}^{(3)}(t)=0, \quad v_{k}^{(3)}\left(t_{k-1}\right)=v_{k}^{(2)}\left(t_{k}\right), \\
& \frac{\mathrm{d} v_{k}^{(4)}(t)}{\mathrm{d} t}+\frac{1}{2} A_{2} v_{k}^{(4)}(t)=0, \quad v_{k}^{(4)}\left(t_{k-1}\right)=v_{k}^{(3)}\left(t_{k}\right), \\
& \frac{\mathrm{d} v_{k}^{(5)}(t)}{\mathrm{d} t}+\frac{\alpha}{2} A_{1} v_{k}^{(5)}(t)=0, \quad v_{k}^{(5)}\left(t_{k-1}\right)=v_{k}^{(4)}\left(t_{k}\right), \\
& \frac{\mathrm{d} w_{k}^{(1)}(t)}{\mathrm{d} t}+\frac{\alpha}{2} A_{2} w_{k}^{(1)}(t)=0, \quad w_{k}^{(1)}\left(t_{k-1}\right)=u_{k-1}\left(t_{k-1}\right), \\
& \frac{\mathrm{d} w_{k}^{(2)}(t)}{\mathrm{d} t}+\frac{1}{2} A_{1} w_{k}^{(2)}(t)=0, \quad w_{k}^{(2)}\left(t_{k-1}\right)=w_{k}^{(1)}\left(t_{k}\right), \\
& \frac{\mathrm{d} w_{k}^{(3)}(t)}{\mathrm{d} t}+\bar{\alpha} A_{2} w_{k}^{(3)}(t)=0, \quad w_{k}^{(3)}\left(t_{k-1}\right)=w_{k}^{(2)}\left(t_{k}\right), \\
& \frac{\mathrm{d} w_{k}^{(4)}(t)}{\mathrm{d} t}+\frac{1}{2} A_{1} w_{k}^{(4)}(t)=0, \quad w_{k}^{(4)}\left(t_{k-1}\right)=w_{k}^{(3)}\left(t_{k}\right), \\
& \frac{\mathrm{d} w_{k}^{(5)}(t)}{\mathrm{d} t}+\frac{\alpha}{2} A_{2} w_{k}^{(5)}(t)=0, \quad w_{k}^{(5)}\left(t_{k-1}\right)=w_{k}^{(4)}\left(t_{k}\right),
\end{aligned}
$$

where $\alpha$ is a complex number with the positive real part, $\operatorname{Re}(\alpha)>0 ; u_{0}(0)=\varphi$. Suppose that the operators $\left(-A_{j}\right),\left(-\alpha A_{j}\right),\left(-\bar{\alpha} A_{j}\right), j=1,2$ generate strongly continuous semigroups.

$u_{k}(t), k=1,2, . .$, is defined on each interval $\left[t_{k-1}, t_{k}\right]$ as follows:

$$
u_{k}(t)=\frac{1}{2}\left[v_{k}^{(5)}(t)+w_{k}^{(5)}(t)\right] .
$$


We declare function $u_{k}(t)$ as an approximated solution of problem (1) on each interval $\left[t_{k-1}, t_{k}\right]$.

\section{Estimate of ERROR OF THE APPROXIMATED SOLUtion}

We need the natural powers $\left(A^{s}, \quad s=2,3,4,5\right)$ of the operator $A=A_{1}+A_{2}$. They are usually defined as follows:

$$
\begin{aligned}
A^{2}= & \left(A_{1}^{2}+A_{2}^{2}\right)+\left(A_{1} A_{2}+A_{2} A_{1}\right), \\
A^{3}= & \left(A_{1}^{3}+A_{2}^{3}\right)+\left(A_{1}^{2} A_{2}+\ldots+A_{2}^{2} A_{1}\right)+\left(A_{1} A_{2} A_{1}+A_{2} A_{1} A_{2}\right), \\
A^{4}= & \left(A_{1}^{4}+A_{2}^{4}\right)+\left(A_{1}^{3} A_{2}+\ldots+A_{2}^{3} A_{1}\right)+\left(A_{1}^{2} A_{2} A_{1}+\ldots+A_{2}^{2} A_{1} A_{2}\right) \\
& +\left(A_{1} A_{2} A_{1} A_{2}+A_{2} A_{1} A_{2} A_{1}\right), \\
A^{5}= & \left(A_{1}^{5}+A_{2}^{5}\right)+\left(A_{1}^{4} A_{2}+\ldots+A_{2}^{4} A_{1}\right)+\left(A_{1}^{4} A_{2} A_{1}+\ldots+A_{2}^{4} A_{1} A_{2}\right) \\
& +\left(A_{1}^{2} A_{2} A_{1} A_{2}+A_{2}^{2} A_{1} A_{2} A_{1}\right)+\left(A_{1} A_{2} A_{1} A_{2} A_{1}+A_{2} A_{1} A_{2} A_{1} A_{2}\right) .
\end{aligned}
$$

It is obvious that the definition domain $D\left(A^{s}\right)$ of the operator $A^{s}$ represents an intersection of definition domains of its addends.

Let us introduce the following notations:

$$
\begin{aligned}
\|\varphi\|_{A} & =\left\|A_{1} \varphi\right\|+\left\|A_{2} \varphi\right\|, \quad \varphi \in D(A) ; \\
\|\varphi\|_{A^{2}} & =\left\|A_{1}^{2} \varphi\right\|+\left\|A_{2}^{2} \varphi\right\|+\left\|A_{1} A_{2} \varphi\right\|+\left\|A_{2} A_{1} \varphi\right\|, \quad \varphi \in D\left(A^{2}\right),
\end{aligned}
$$

where $\|\cdot\|$ is a norm in $X .\|\varphi\|_{A^{s}}, \quad(s=3,4,5)$ is defined analogously.

Theorem. Let the following conditions be fulfilled:

(a) $\alpha=\frac{1}{2} \pm i \frac{1}{2 \sqrt{3}} \quad(i=\sqrt{-1})$;

(b) Operators $\left(-\gamma A_{j}\right), \gamma=1, \alpha, \bar{\alpha}(j=1,2)$ and $(-A)$ generate strongly continuous semigroups, for which the following estimates are true:

$$
\begin{aligned}
\left\|U\left(t, \gamma A_{j}\right)\right\| & \leq \mathrm{e}^{\omega t} \\
\|U(t, A)\| & \leq M \mathrm{e}^{\omega t}, \quad M=\text { const. }>0
\end{aligned}
$$

(c) $U(s, A) \varphi \in D\left(A^{5}\right)$ for each fixed $s \geq 0$.

Then the following estimate holds:

$$
\left\|u\left(t_{k}\right)-u_{k}\left(t_{k}\right)\right\| \leq c \mathrm{e}^{\omega_{0} t_{k}} t_{k} \tau^{4} \sup _{s \in\left[0, t_{k}\right]}\|U(s, A) \varphi\|_{A^{5}},
$$

where $c$ and $\omega_{0}$ are positive constants.

Proof. According to the following formula (see [32], p. 603):

$$
A \int_{r}^{t} U(s, A) \mathrm{d} s=U(r, A)-U(t, A), \quad 0 \leq r \leq t,
$$

we can obtain the expansion:

$$
U(t, A)=\sum_{i=0}^{k-1}(-1)^{i} \frac{t^{i}}{i !} A^{i}+R_{k}(t, A)
$$

where

$$
R_{k}(t, A)=(-A)^{k} \int_{0}^{t} \int_{0}^{s_{1}} \ldots \int_{0}^{s_{k-1}} U(s, A) \mathrm{d} s \mathrm{~d} s_{k-1} \ldots \mathrm{d} s_{1} .
$$


From formula (3) we obtain:

$$
u_{k}\left(t_{k}\right)=V^{k}(\tau) \varphi
$$

where

and where

$$
V(\tau)=\frac{1}{2}\left[V_{1}(\tau)+V_{2}(\tau)\right]
$$

$$
\begin{aligned}
& V_{1}(\tau)=U\left(\tau, \frac{\alpha}{2} A_{1}\right) U\left(\tau, \frac{1}{2} A_{2}\right) U\left(\tau, \bar{\alpha} A_{1}\right) U\left(\tau, \frac{1}{2} A_{2}\right) U\left(\tau, \frac{\alpha}{2} A_{1}\right), \\
& V_{2}(\tau)=U\left(\tau, \frac{\alpha}{2} A_{2}\right) U\left(\tau, \frac{1}{2} A_{1}\right) U\left(\tau, \bar{\alpha} A_{2}\right) U\left(\tau, \frac{1}{2} A_{1}\right) U\left(\tau, \frac{\alpha}{2} A_{2}\right) .
\end{aligned}
$$

Remark 1. Stability of the considered scheme on each finite time interval follows from the first inequality of the condition (b) of the theorem. In this case, for the solving operator, the following estimate holds:

$$
\left\|V^{k}(\tau)\right\| \leq \mathrm{e}^{\omega_{1} t_{k}}
$$

where $\omega_{1}$ is a positive constant.

We introduce the following notations for combinations (sum, product) of semigroups. Let $T(\tau)$ be a combination (sum, product) of the semigroups, which are generated by the operators $\left(-\gamma A_{i}\right)(i=1,2)$. Let us decompose every semigroup included in operator $T(\tau)$ according to formula (4), multiply these decompositions on each other, add the similar members and, in the decomposition thus obtained, denote coefficients of the members $\left(-\tau A_{i}\right),\left(\tau^{2} A_{i} A_{j}\right),\left(-\tau^{3} A_{i} A_{j} A_{k}\right)$ and $\left(\tau^{4} A_{i} A_{j} A_{k} A_{l}\right)(i, j, k, l=1,2)$ respectively by $[T(\tau)]_{i}$, $[T(\tau)]_{i, j},[T(\tau)]_{i, j, k}$ and $[T(\tau)]_{i, j, k, l}$.

If we decompose all the semigroups included in the operator $V(\tau)$ according to formula (4) from left to right in such a way that each residual term appears of the fifth order, we will obtain the following formula:

$$
\begin{aligned}
V(\tau)= & I-\tau \sum_{i=1}^{2}[V(\tau)]_{i} A_{i}+\tau^{2} \sum_{i, j=1}^{2}[V(\tau)]_{i, j} A_{i} A_{j}-\tau^{3} \sum_{i, j, k=1}^{2}[V(\tau)]_{i, j, k} A_{i} A_{j} A_{k} \\
& +\tau^{4} \sum_{i, j, k, l=1}^{2}[V(\tau)]_{i, j, k, l} A_{i} A_{j} A_{k} A_{l}+R_{5}(\tau) .
\end{aligned}
$$

According to the first inequality of the condition (b) of the Theorem, for $R_{5}(\tau)$, the following estimate holds:

$$
\left\|R_{5}(\tau) \varphi\right\| \leq c \mathrm{e}^{\omega_{0} \tau} \tau^{5}\|\varphi\|_{A^{5}}, \quad \varphi \in D\left(A^{5}\right)
$$

where $c$ and $\omega_{0}$ are positive constants.

It is obvious that, for the coefficients in formula (8), we have:

$$
\begin{aligned}
{[V(\tau)]_{i} } & =\frac{1}{2}\left(\left[V_{1}(\tau)\right]_{i}+\left[V_{2}(\tau)\right]_{i}\right), \quad i=1,2, \\
{[V(\tau)]_{i, j} } & =\frac{1}{2}\left(\left[V_{1}(\tau)\right]_{i, j}+\left[V_{2}(\tau)\right]_{i, j}\right), \quad i, j=1,2, \\
{[V(\tau)]_{i, j, k} } & =\frac{1}{2}\left(\left[V_{1}(\tau)\right]_{i, j, k}+\left[V_{2}(\tau)\right]_{i, j, k}\right), \quad i, j, k=1,2, \\
{[V(\tau)]_{i, j, k, l} } & =\frac{1}{2}\left(\left[V_{1}(\tau)\right]_{i, j, k, l}+\left[V_{2}(\tau)\right]_{i, j, k, l}\right), \quad i, j, k, l=1,2 .
\end{aligned}
$$

Let us make two remarks which will simplify a calculation of coefficients in decomposition (8): 
Remark 2. Operator $V(\tau)$ will not change if we replace with each other the operators $A_{1}$ and $A_{2}$ in its expression, as in this case $V_{1}(\tau)$ will coincide with $V_{2}(\tau)$, and $V_{2}(\tau)$ - with $V_{1}(\tau)$. Therefore we have:

$$
\begin{aligned}
{[V(\tau)]_{i} } & =[V(\tau)]_{3-i}, \quad i=1,2 \\
{[V(\tau)]_{i, j} } & =[V(\tau)]_{3-i, 3-j}, \quad i, j=1,2 \\
{[V(\tau)]_{i, j, k} } & =[V(\tau)]_{3-i, 3-j, 3-k}, \quad i, j, k=1,2 \\
{[V(\tau)]_{i, j, k, l} } & =[V(\tau)]_{3-i, 3-j, 3-k, 3-l}, \quad i, j, k, l=1,2 .
\end{aligned}
$$

Remark 3. Operators $V_{1}(\tau)$ and $V_{2}(\tau)$ are symmetrical in the sense that in their expressions the factors (semigroups) equally remote from the ends coincide with each other. Therefore we have:

$$
\begin{aligned}
{[V(\tau)]_{i, j} } & =[V(\tau)]_{j, i}, \quad i, j=1,2 \\
{[V(\tau)]_{i, j, k} } & =[V(\tau)]_{k, j, i}, \quad i, j, k=1,2 \\
{[V(\tau)]_{i, j, k, l} } & =[V(\tau)]_{l, k, j, i}, \quad i, j, k, l=1,2 .
\end{aligned}
$$

Let us calculate the coefficients $[V(\tau)]_{i}$ corresponding to the first order members in formula (8). It is obvious that the members, corresponding to these coefficients, are obtained from the decomposition of only those factors (semigroups) of the operator $V(\tau)$, which are generated by the operators $\left(-\gamma A_{i}\right)$, and from the decomposition of other semigroups only first addends (the members with identical operators) will participate.

On the whole, we have two cases: $i=1$ and $i=2$. Let us consider the case $i=1$. We obviously have:

$$
\left[V_{1}(\tau)\right]_{1}=\left[U\left(\tau, A_{1}\right)\right]_{1}=1
$$

and

Thus

$$
\left[V_{2}(\tau)\right]_{1}=\left[U\left(\tau, A_{1}\right)\right]_{1}=1 .
$$

According to Remark 2:

$$
[V(\tau)]_{1}=\frac{1}{2}\left(\left[V_{1}(\tau)\right]_{1}+\left[V_{2}(\tau)\right]_{1}\right)=1 .
$$

$$
[V(\tau)]_{2}=[V(\tau)]_{1}=1 .
$$

Let us calculate the coefficients $[V(\tau)]_{i, j}(i, j=1,2)$ corresponding to the second order members included in formula (8). On the whole we have two cases: $(i, j)=(1,1),(1,2),(2,1),(2,2)$. Let us consider the case $(i, j)=(1,1)$. We obviously have:

and

$$
\left[V_{1}(\tau)\right]_{1,1}=\left[U\left(\tau, A_{1}\right)\right]_{1,1}=\frac{1}{2}
$$

Therefore

$$
\left[V_{2}(\tau)\right]_{1,1}=\left[U\left(\tau, A_{1}\right)\right]_{1,1}=\frac{1}{2}
$$

According to Remark 2:

$$
[V(\tau)]_{1,1}=\frac{1}{2}\left(\left[V_{1}(\tau)\right]_{1,1}+\left[V_{2}(\tau)\right]_{1,1}\right)=\frac{1}{2} .
$$

$$
[V(\tau)]_{2,2}=[V(\tau)]_{1,1}=\frac{1}{2}
$$

Let us consider the case $(i, j)=(1,2)$, we obviously have:

$$
\begin{aligned}
{\left[V_{1}(\tau)\right]_{1,2}=\left[U\left(\tau, \frac{\alpha}{2} A_{1}\right)\right]_{1}\left[U\left(\tau, \frac{1}{2} A_{2}\right)\right]_{2}+\left[U\left(\tau, \frac{\alpha}{2} A_{1}\right)\right]_{1}\left[U\left(\tau, \frac{1}{2} A_{2}\right)\right]_{2} } & +\left[U\left(\tau, \bar{\alpha} A_{1}\right)\right]_{1}\left[U\left(\tau, \frac{1}{2} A_{2}\right)\right]_{2}=\frac{\alpha}{2} \frac{1}{2}+\frac{\alpha}{2} \frac{1}{2}+\bar{\alpha} \frac{1}{2}=\frac{1}{2}
\end{aligned}
$$


and

$$
\begin{aligned}
{\left[V_{2}(\tau)\right]_{1,2}=\left[U\left(\tau, \frac{1}{2} A_{1}\right)\right]_{1}\left[U\left(\tau, \bar{\alpha} A_{2}\right)\right]_{2}+\left[U\left(\tau, \frac{1}{2} A_{1}\right)\right]_{1}\left[U\left(\tau, \frac{\alpha}{2} A_{2}\right)\right]_{2} } \\
+\left[U\left(\tau, \frac{1}{2} A_{1}\right)\right]_{1}\left[U\left(\tau, \frac{\alpha}{2} A_{2}\right)\right]_{2}=\bar{\alpha} \frac{1}{2}+\frac{\alpha}{2} \frac{1}{2}+\frac{\alpha}{2} \frac{1}{2}=\frac{1}{2}
\end{aligned}
$$

Thus

According to Remark 2:

$$
[V(\tau)]_{1,2}=\frac{1}{2}\left(\left[V_{1}(\tau)\right]_{1,2}+\left[V_{2}(\tau)\right]_{1,2}\right)=\frac{1}{2}
$$

$$
[V(\tau)]_{2,1}=[V(\tau)]_{1,2}=\frac{1}{2}
$$

Here we used the identity $\alpha+\bar{\alpha}=1$.

By combining formulas (11) and (12), we will obtain:

$$
[V(\tau)]_{i, j}=\frac{1}{2}, \quad i, j=1,2 .
$$

Let us calculate the coefficients $[V(\tau)]_{i, j, k}(i, j, k=1,2)$ corresponding to the third order members in formula (8). On the whole we have eight cases: $(i, j, k)=(1,1,1),(1,1,2),(1,2,1),(1,2,2),(2,1,1),(2,1,2)$, $(2,2,1),(2,2,2)$. Let us consider the case $(i, j, k)=(1,1,1)$. We obviously have:

$$
\left[V_{1}(\tau)\right]_{1,1,1}=\left[U\left(\tau, A_{1}\right)\right]_{1,1,1}=\frac{1}{6}
$$

and

Thus:

$$
\left[V_{2}(\tau)\right]_{1,1,1}=\left[U\left(\tau, A_{1}\right)\right]_{1,1,1}=\frac{1}{6}
$$

According to Remark 2:

$$
[V(\tau)]_{1,1,1}=\frac{1}{2}\left(\left[V_{1}(\tau)\right]_{1,1,1}+\left[V_{2}(\tau)\right]_{1,1,1}\right)=\frac{1}{6}
$$

$$
[V(\tau)]_{2,2,2}=[V(\tau)]_{1,1,1}=\frac{1}{6}
$$

Let us calculate the case $(i, j, k)=(1,1,2)$. We obviously have:

$$
\begin{aligned}
{\left[V_{1}(\tau)\right]_{1,1,2}=} & {\left[U\left(\tau, \frac{\alpha}{2} A_{1}\right)\right]_{1,1}\left[U\left(\tau, \frac{1}{2} A_{2}\right)\right]_{2}+\left[U\left(\tau, \frac{\alpha}{2} A_{1}\right)\right]_{1,1}\left[U\left(\tau, \frac{1}{2} A_{2}\right)\right]_{2} } \\
& +\left[U\left(\tau, \frac{\alpha}{2} A_{1}\right)\right]_{1}\left[U\left(\tau, \bar{\alpha} A_{1}\right)\right]_{1}\left[U\left(\tau, \frac{1}{2} A_{2}\right)\right]_{2}+\left[U\left(\tau, \bar{\alpha} A_{1}\right)\right]_{1,1}\left[U\left(\tau, \frac{1}{2} A_{2}\right)\right]_{2} \\
= & \frac{1}{2} \frac{\alpha^{2}}{8}+\frac{1}{2} \frac{\alpha^{2}}{8}+\frac{\alpha}{2} \bar{\alpha} \frac{1}{2}+\frac{\bar{\alpha}^{2}}{2} \frac{1}{2}=\frac{1+\bar{\alpha}^{2}}{8}
\end{aligned}
$$

and

$$
\begin{aligned}
{\left[V_{2}(\tau)\right]_{1,1,2}=} & {\left[U\left(\tau, \frac{1}{2} A_{1}\right)\right]_{1,1}\left[U\left(\tau, \bar{\alpha} A_{2}\right)\right]_{2}+\left[U\left(\tau, \frac{1}{2} A_{1}\right)\right]_{1,1}\left[U\left(\tau, \frac{\alpha}{2} A_{2}\right)\right]_{2} } \\
& +\left[U\left(\tau, \frac{1}{2} A_{1}\right)\right]_{1}\left[U\left(\tau, \frac{1}{2} A_{1}\right)\right]_{1}\left[U\left(\tau, \frac{\alpha}{2} A_{2}\right)\right]_{2}++\left[U\left(\tau, \frac{1}{2} A_{1}\right)\right]_{1,1}\left[U\left(\tau, \frac{\alpha}{2} A_{2}\right)\right]_{2} \\
= & \frac{1}{8} \bar{\alpha}+\frac{1}{8} \frac{\alpha}{2}+\frac{1}{2} \frac{1}{2} \frac{\alpha}{2}+\frac{1}{8} \frac{\alpha}{2}=\frac{1+\alpha}{8}
\end{aligned}
$$


Thus

$$
[V(\tau)]_{1,1,2}=\frac{1}{2}\left(\left[V_{1}(\tau)\right]_{1,1,2}+\left[V_{2}(\tau)\right]_{1,1,2}\right)=\frac{2+\bar{\alpha}^{2}+\alpha}{16}=\frac{1}{6}
$$

Here we used the identities $\alpha+\bar{\alpha}=1, \alpha \bar{\alpha}=\frac{1}{3}$ and $\alpha+\bar{\alpha}^{2}=\frac{2}{3}$.

According to Remarks 2 and 3 :

$$
[V(\tau)]_{1,1,2}=[V(\tau)]_{2,1,1}=[V(\tau)]_{2,2,1}=[V(\tau)]_{1,2,2}=\frac{1}{6}
$$

Let us consider the case $(i, j, k)=(1,2,1)$. We obviously have:

$$
\begin{aligned}
{\left[V_{1}(\tau)\right]_{1,2,1}=} & {\left[U\left(\tau, \frac{\alpha}{2} A_{1}\right)\right]_{1}\left[U\left(\tau, \frac{1}{2} A_{2}\right)\right]_{2}\left[U\left(\tau, \bar{\alpha} A_{1}\right)\right]_{1} } \\
& +\left[U\left(\tau, \frac{\alpha}{2} A_{1}\right)\right]_{1}\left[U\left(\tau, \frac{1}{2} A_{2}\right)\right]_{2}\left[U\left(\tau, \frac{\alpha}{2} A_{1}\right)\right]_{1} \\
& +\left[U\left(\tau, \frac{\alpha}{2} A_{1}\right)\right]_{1}\left[U\left(\tau, \frac{1}{2} A_{2}\right)\right]_{2}\left[U\left(\tau, \frac{\alpha}{2} A_{1}\right)\right]_{1} \\
& +\left[U\left(\tau, \bar{\alpha} A_{1}\right)\right]_{1}\left[U\left(\tau, \frac{1}{2} A_{2}\right)\right]_{2}\left[U\left(\tau, \frac{\alpha}{2} A_{1}\right)\right]_{1} \\
= & \frac{\alpha}{2} \frac{1}{2} \bar{\alpha}+\frac{\alpha}{2} \frac{1}{2} \frac{\alpha}{2}+\frac{\alpha}{2} \frac{1}{2} \frac{\alpha}{2}+\bar{\alpha} \frac{1}{2} \frac{\alpha}{2}=\frac{1}{6}+\frac{\alpha^{2}}{4}
\end{aligned}
$$

and

$$
\left[V_{2}(\tau)\right]_{1,2,1}=\left[U\left(\tau, \frac{1}{2} A_{1}\right)\right]_{1}\left[U\left(\tau, \bar{\alpha} A_{2}\right)\right]_{2}\left[U\left(\tau, \frac{1}{2} A_{1}\right)\right]_{1}=\frac{\bar{\alpha}}{4}
$$

Thus

$$
[V(\tau)]_{1,2,1}=\frac{1}{2}\left(\left[V_{1}(\tau)\right]_{1,1,2}+\left[V_{2}(\tau)\right]_{1,1,2}\right)=\frac{1}{12}+\frac{\alpha^{2}+\bar{\alpha}}{8}=\frac{1}{6} .
$$

Here we used the identity $\alpha^{2}+\bar{\alpha}=\frac{2}{3}$.

According to Remark 2:

$$
[V(\tau)]_{2,1,2}=[V(\tau)]_{1,2,1}=\frac{1}{6}
$$

By combining formulas (14), (15) and (16), we will obtain:

$$
[V(\tau)]_{i, j, k}=\frac{1}{6}, \quad i, j, k=1,2 .
$$

Let us calculate the coefficients $[V(\tau)]_{i, j, k, l}(i, j, k, l=1,2)$ corresponding to the fourth order members in formula (8). On the whole we have sixteen cases: $(i, j, k, l)=(1,1,1,1),(1,1,1,2), \ldots,(2,2,2,1),(2,2,2,2)$. Let us consider the case $(i, j, k, l)=(1,1,1,1)$. We obviously have:

$$
\left[V_{1}(\tau)\right]_{1,1,1,1}=\left[U\left(\tau, A_{1}\right)\right]_{1,1,1,1}=\frac{1}{24}
$$

and

Thus:

$$
\left[V_{2}(\tau)\right]_{1,1,1,1}=\left[U\left(\tau, A_{1}\right)\right]_{1,1,1,1}=\frac{1}{24}
$$

$$
[V(\tau)]_{1,1,1,1}=\frac{1}{2}\left(\left[V_{1}(\tau)\right]_{1,1,1,1}+\left[V_{2}(\tau)\right]_{1,1,1,1}\right)=\frac{1}{24}
$$


According to Remark 2:

$$
[V(\tau)]_{2,2,2,2}=[V(\tau)]_{1,1,1,1}=\frac{1}{24} .
$$

Let us consider the case $(i, j, k, l)=(1,1,1,2)$, we obviously have:

$$
\begin{aligned}
{\left[V_{1}(\tau)\right]_{1,1,1,2}=} & {\left[U\left(\tau, \frac{\alpha}{2} A_{1}\right)\right]_{1,1,1}\left[U\left(\tau, \frac{1}{2} A_{2}\right)\right]_{2}+\left[U\left(\tau, \frac{\alpha}{2} A_{1}\right)\right]_{1,1,1}\left[U\left(\tau, \frac{1}{2} A_{2}\right)\right]_{2} } \\
& +\left[U\left(\tau, \frac{\alpha}{2} A_{1}\right)\right]_{1,1}\left[U\left(\tau, \bar{\alpha} A_{1}\right)\right]_{1}\left[U\left(\tau, \frac{1}{2} A_{2}\right)\right]_{2}+\left[U\left(\tau, \frac{\alpha}{2} A_{1}\right)\right]_{1}\left[U\left(\tau, \bar{\alpha} A_{1}\right)\right]_{1,1}\left[U\left(\tau, \frac{1}{2} A_{2}\right)\right]_{2} \\
& +\left[U\left(\tau, \bar{\alpha} A_{1}\right)\right]_{1,1,1}\left[U\left(\tau, \frac{1}{2} A_{2}\right)\right]_{2} \frac{\alpha^{3}}{48} \frac{1}{2}+\frac{\alpha^{3}}{48} \frac{1}{2}+\frac{\alpha^{2}}{8} \bar{\alpha} \frac{1}{2}+\frac{\alpha}{2} \frac{\bar{\alpha}^{2}}{2} \frac{1}{2}+\frac{\bar{\alpha}^{3}}{6} \frac{1}{2} \\
= & \frac{\alpha^{3}+\alpha+4 \bar{\alpha}^{3}+2 \bar{\alpha}}{48}=\frac{1+3 \bar{\alpha}^{3}+\bar{\alpha}}{48}
\end{aligned}
$$

and

$$
\begin{aligned}
{\left[V_{2}(\tau)\right]_{1,1,1,2}=} & {\left[U\left(\tau, \frac{1}{2} A_{1}\right)\right]_{1,1,1}\left[U\left(\tau, \bar{\alpha} A_{2}\right)\right]_{2}+\left[U\left(\tau, \frac{1}{2} A_{1}\right)\right]_{1,1,1}\left[U\left(\tau, \frac{\alpha}{2} A_{2}\right)\right]_{2} } \\
& +\left[U\left(\tau, \frac{1}{2} A_{1}\right)\right]_{1,1}\left[U\left(\tau, \frac{1}{2} A_{1}\right)\right]_{1}\left[U\left(\tau, \frac{\alpha}{2} A_{2}\right)\right]_{2} \\
& +\left[U\left(\tau, \frac{1}{2} A_{1}\right)\right]_{1}\left[U\left(\tau, \frac{1}{2} A_{1}\right)\right]_{1,1}\left[U\left(\tau, \frac{\alpha}{2} A_{2}\right)\right]_{2}+\left[U\left(\tau, \frac{1}{2} A_{1}\right)\right]_{1,1,1}\left[U\left(\tau, \frac{\alpha}{2} A_{2}\right)\right]_{2} \\
= & \frac{1}{48} \bar{\alpha}+\frac{1}{48} \frac{\alpha}{2}+\frac{1}{8} \frac{1}{2} \frac{\alpha}{2}+\frac{1}{2} \frac{1}{8} \frac{\alpha}{2}+\frac{1}{48} \frac{\alpha}{2}=\frac{1+3 \alpha}{48} .
\end{aligned}
$$

Thus

$$
[V(\tau)]_{1,1,1,2}=\frac{1}{2}\left(\left[V_{1}(\tau)\right]_{1,1,1,2}+\left[V_{2}(\tau)\right]_{1,1,1,2}\right)=\frac{2+3\left(\bar{\alpha}^{3}+\alpha\right)+\bar{\alpha}}{96}=\frac{1}{24} .
$$

Here we used the identities $3\left(\bar{\alpha}^{3}+\alpha\right)=2-\bar{\alpha}, \quad \bar{\alpha}^{3}+\alpha^{3}=0$.

According to Remarks 2 and 3 :

$$
[V(\tau)]_{1,1,1,2}=[V(\tau)]_{2,1,1,1}=[V(\tau)]_{1,2,2,2}=[V(\tau)]_{2,2,2,1}=\frac{1}{24}
$$

Let us consider the case $(i, j, k, l)=(1,1,2,1)$. We obviously have:

$$
\begin{aligned}
{\left[V_{1}(\tau)\right]_{1,1,2,1}=} & {\left[U\left(\tau, \frac{\alpha}{2} A_{1}\right)\right]_{1,1}\left[U\left(\tau, \frac{1}{2} A_{2}\right)\right]_{2}\left[U\left(\tau, \bar{\alpha} A_{1}\right)\right]_{1}+\left[U\left(\tau, \frac{\alpha}{2} A_{1}\right)\right]_{1,1}\left[U\left(\tau, \frac{1}{2} A_{2}\right)\right]_{2}\left[U\left(\tau, \frac{\alpha}{2} A_{1}\right)\right]_{1} } \\
& +\left[U\left(\tau, \frac{\alpha}{2} A_{1}\right)\right]_{1,1}\left[U\left(\tau, \frac{1}{2} A_{2}\right)\right]_{2}\left[U\left(\tau, \frac{\alpha}{2} A_{1}\right)\right]_{1} \\
& +\left[U\left(\tau, \frac{\alpha}{2} A_{1}\right)\right]_{1}\left[U\left(\tau, \bar{\alpha} A_{1}\right)\right]_{1}\left[U\left(\tau, \frac{1}{2} A_{2}\right)\right]_{2}\left[U\left(\tau, \frac{\alpha}{2} A_{1}\right)\right]_{1} \\
& +\left[U\left(\tau, \bar{\alpha} A_{1}\right)\right]_{1,1}\left[U\left(\tau, \frac{1}{2} A_{2}\right)\right]_{2}\left[U\left(\tau, \frac{\alpha}{2} A_{1}\right)\right]_{1} \\
= & \frac{\alpha^{2}}{8} \frac{1}{2} \bar{\alpha}+\frac{\alpha^{2}}{8} \frac{1}{2} \frac{\alpha}{2}+\frac{\alpha^{2}}{8} \frac{1}{2} \frac{\alpha}{2}+\frac{\alpha}{2} \bar{\alpha} \frac{1}{2} \frac{\alpha}{2}+\frac{\bar{\alpha}^{2}}{2} \frac{1}{2} \frac{\alpha}{2} \\
= & \frac{3 \alpha^{3}+3 \alpha+2 \bar{\alpha}}{48}=\frac{3 \alpha^{3}+\alpha+2}{48}
\end{aligned}
$$


and

$$
\left[V_{2}(\tau)\right]_{1,1,2,1}=\left[U\left(\tau, \frac{1}{2} A_{1}\right)\right]_{1,1}\left[U\left(\tau, \bar{\alpha} A_{2}\right)\right]_{2}\left[U\left(\tau, \frac{1}{2} A_{1}\right)\right]_{1}=\frac{1}{8} \bar{\alpha} \frac{1}{2}=\frac{1}{16} \bar{\alpha}
$$

Thus

$$
[V(\tau)]_{1,1,2,1}=\frac{1}{2}\left(\left[V_{1}(\tau)\right]_{1,1,2,1}+\left[V_{2}(\tau)\right]_{1,1,2,1}\right)=\frac{3 \alpha^{3}+\alpha+2+3 \bar{\alpha}}{96}=\frac{3\left(\alpha^{3}+\bar{\alpha}\right)+\alpha+2}{96}=\frac{1}{24} .
$$

Here we used the identity $\bar{\alpha}^{3}+\alpha^{3}=0$.

According to Remarks 2 and 3:

$$
[V(\tau)]_{1,1,2,1}=[V(\tau)]_{2,2,1,2}=[V(\tau)]_{1,2,1,1}=[V(\tau)]_{2,1,2,2}=\frac{1}{24}
$$

Let us consider the case $(i, j, k, l)=(1,1,2,2)$. We obviously have:

$$
\begin{aligned}
{\left[V_{1}(\tau)\right]_{1,1,2,2}=} & {\left[U\left(\tau, \frac{\alpha}{2} A_{1}\right)\right]_{1,1}\left[U\left(\tau, \frac{1}{2} A_{2}\right)\right]_{2,2}+\left[U\left(\tau, \frac{\alpha}{2} A_{1}\right)\right]_{1,1}\left[U\left(\tau, \frac{1}{2} A_{2}\right)\right]_{2}\left[U\left(\tau, \frac{1}{2} A_{2}\right)\right]_{2} } \\
& +\left[U\left(\tau, \frac{\alpha}{2} A_{1}\right)\right]_{1,1}\left[U\left(\tau, \frac{1}{2} A_{2}\right)\right]_{2,2}+\left[U\left(\tau, \frac{\alpha}{2} A_{1}\right)\right]_{1}\left[U\left(\tau, \bar{\alpha} A_{1}\right)\right]_{1}\left[U\left(\tau, \frac{1}{2} A_{2}\right)\right]_{2,2} \\
& +\left[U\left(\tau, \bar{\alpha} A_{1}\right)\right]_{1,1}\left[U\left(\tau, \frac{1}{2} A_{2}\right)\right]_{2,2} \\
= & \frac{\alpha^{2}}{8} \frac{1}{8}+\frac{\alpha^{2}}{8} \frac{1}{2} \frac{1}{2}+\frac{\alpha^{2}}{8} \frac{1}{8}+\frac{\alpha}{2} \bar{\alpha} \frac{1}{8}+\frac{\bar{\alpha}^{2}}{2} \frac{1}{8} \\
= & \frac{\alpha^{2}+\alpha \bar{\alpha}+\bar{\alpha}^{2}}{16}=\frac{1}{24}
\end{aligned}
$$

and

$$
\begin{aligned}
{\left[V_{2}(\tau)\right]_{1,1,2,2}=} & {\left[U\left(\tau, \frac{1}{2} A_{1}\right)\right]_{1,1}\left[U\left(\tau, \bar{\alpha} A_{2}\right)\right]_{2,2}\left[U\left(\tau, \frac{1}{2} A_{1}\right)\right]_{1,1}\left[U\left(\tau, \bar{\alpha} A_{2}\right)\right]_{2}\left[U\left(\tau, \frac{\alpha}{2} A_{2}\right)\right]_{2} } \\
& +\left[U\left(\tau, \frac{1}{2} A_{1}\right)\right]_{1,1}\left[U\left(\tau, \frac{\alpha}{2} A_{2}\right)\right]_{2,2}+\left[U\left(\tau, \frac{1}{2} A_{1}\right)\right]_{1}\left[U\left(\tau, \frac{1}{2} A_{1}\right)\right]_{1}\left[U\left(\tau, \frac{\alpha}{2} A_{2}\right)\right]_{2,2} \\
& +\left[U\left(\tau, \frac{1}{2} A_{1}\right)\right]_{1,1}\left[U\left(\tau, \frac{\alpha}{2} A_{2}\right)\right]_{2,2} \\
= & \frac{1}{8} \frac{\bar{\alpha}^{2}}{2}+\frac{1}{8} \bar{\alpha} \frac{\alpha}{2}+\frac{1}{8} \frac{\alpha^{2}}{8}+\frac{1}{2} \frac{1}{2} \frac{\alpha^{2}}{8}+\frac{1}{8} \frac{\alpha^{2}}{8} \\
= & \frac{\bar{\alpha}^{2}+\bar{\alpha} \alpha+\alpha^{2}}{16}=\frac{1}{24} .
\end{aligned}
$$

Thus

$$
[V(\tau)]_{1,1,2,2}=\frac{1}{2}\left(\left[V_{1}(\tau)\right]_{1,1,2,2}+\left[V_{2}(\tau)\right]_{1,1,2,2}\right)=\frac{1}{24}
$$

According to Remark 2:

$$
[V(\tau)]_{1,1,2,2}=[V(\tau)]_{2,2,1,1}=\frac{1}{24}
$$


Let us consider the case $(i, j, k, l)=(1,2,2,1)$. We obviously have:

$$
\begin{aligned}
{\left[V_{1}(\tau)\right]_{1,2,2,1}=} & {\left[U\left(\tau, \frac{\alpha}{2} A_{1}\right)\right]_{1}\left[U\left(\tau, \frac{1}{2} A_{2}\right)\right]_{2,2}\left[U\left(\tau, \bar{\alpha} A_{1}\right)\right]_{1} } \\
& +\left[U\left(\tau, \frac{\alpha}{2} A_{1}\right)\right]_{1}\left[U\left(\tau, \frac{1}{2} A_{2}\right)\right]_{2,2}\left[U\left(\tau, \frac{\alpha}{2} A_{1}\right)\right]_{1} \\
& +\left[U\left(\tau, \frac{\alpha}{2} A_{1}\right)\right]_{1}\left[U\left(\tau, \frac{1}{2} A_{2}\right)\right]_{2}\left[U\left(\tau, \frac{1}{2} A_{2}\right)\right]_{2}\left[U\left(\tau, \frac{\alpha}{2} A_{1}\right)\right]_{1} \\
& +\left[U\left(\tau, \frac{\alpha}{2} A_{1}\right)\right]_{1}\left[U\left(\tau, \frac{1}{2} A_{2}\right)\right]_{2,2}\left[U\left(\tau, \frac{\alpha}{2} A_{1}\right)\right]_{1} \\
& +\left[U\left(\tau, \bar{\alpha} A_{1}\right)\right]_{1}\left[U\left(\tau, \frac{1}{2} A_{2}\right)\right]_{2,2}\left[U\left(\tau, \frac{\alpha}{2} A_{1}\right)\right]_{1} \\
= & \frac{\alpha}{2} \frac{1}{8} \bar{\alpha}+\frac{\alpha}{2} \frac{1}{8} \frac{\alpha}{2}+\frac{\alpha}{2} \frac{1}{2} \frac{1}{2} \frac{\alpha}{2}+\frac{\alpha}{2} \frac{1}{8} \frac{\alpha}{2}+\bar{\alpha} \frac{1}{8} \frac{\alpha}{2}=\frac{\alpha^{2}+\alpha \bar{\alpha}}{8}
\end{aligned}
$$

and

$$
\left[V_{2}(\tau)\right]_{1,2,2,1}=\left[U\left(\tau, \frac{1}{2} A_{1}\right)\right]_{1}\left[U\left(\tau, \bar{\alpha} A_{2}\right)\right]_{2,2}\left[U\left(\tau, \frac{1}{2} A_{1}\right)\right]_{1}=\frac{1}{2} \frac{\bar{\alpha}^{2}}{2} \frac{1}{2}=\frac{\bar{\alpha}^{2}}{8}
$$

Thus

$$
[V(\tau)]_{1,2,2,1}=\frac{1}{2}\left(\left[V_{1}(\tau)\right]_{1,2,2,1}+\left[V_{2}(\tau)\right]_{1,2,2,1}\right)=\frac{\alpha^{2}+\alpha \bar{\alpha}+\bar{\alpha}^{2}}{16}=\frac{1}{24} .
$$

According to Remark 2:

$$
[V(\tau)]_{1,2,2,1}=[V(\tau)]_{2,1,1,2}=\frac{1}{24} .
$$

Let us consider the case $(i, j, k, l)=(1,2,1,2)$. We obviously have:

$$
\left[V_{1}(\tau)\right]_{1,2,1,2}=\left[U\left(\tau, \frac{\alpha}{2} A_{1}\right)\right]_{1}\left[U\left(\tau, \frac{1}{2} A_{2}\right)\right]_{2}\left[U\left(\tau, \bar{\alpha} A_{1}\right)\right]_{1}\left[U\left(\tau, \frac{1}{2} A_{2}\right)\right]_{2}=\frac{\alpha}{2} \frac{1}{2} \bar{\alpha} \frac{1}{2}=\frac{1}{24}
$$

and

$$
\left[V_{2}(\tau)\right]_{1,2,1,2}=\left[U\left(\tau, \frac{1}{2} A_{1}\right)\right]_{1}\left[U\left(\tau, \bar{\alpha} A_{2}\right)\right]_{2}\left[U\left(\tau, \frac{1}{2} A_{1}\right)\right]_{1}\left[U\left(\tau, \frac{\alpha}{2} A_{1}\right)\right]_{2}=\frac{1}{2} \bar{\alpha} \frac{1}{2} \frac{\alpha}{2}=\frac{1}{24}
$$

Thus

$$
[V(\tau)]_{1,2,1,2}=\frac{1}{2}\left(\left[V_{1}(\tau)\right]_{1,2,1,2}+\left[V_{2}(\tau)\right]_{1,2,1,2}\right)=\frac{1}{24},
$$

According to Remark 2:

$$
[V(\tau)]_{1,2,1,2}=[V(\tau)]_{2,1,2,1}=\frac{1}{24} .
$$

By combining formulas (18)-(23), we will obtain:

$$
[V(\tau)]_{i, j, k, l}=\frac{1}{24}, \quad i, j, k, l=1,2 .
$$


From equality (8), taking into account formulas (10), (13), (17) and (24), we will obtain:

$$
\begin{aligned}
V(\tau) & =I-\tau \sum_{i=1}^{2} A_{i}+\frac{1}{2} \tau^{2} \sum_{i, j=1}^{2} A_{i} A_{j}-\frac{1}{6} \tau^{3} \sum_{i, j, k=1}^{2} A_{i} A_{j} A_{k}+\frac{1}{24} \tau^{4} \sum_{i, j, k, l=1}^{2} A_{i} A_{j} A_{k} A_{l}+R_{5}(\tau) \\
& =I-\tau \sum_{i=1}^{2} A_{i}+\frac{1}{2} \tau^{2}\left(\sum_{i=1}^{2} A_{i}\right)^{2}-\frac{1}{6} \tau^{3}\left(\sum_{i=1}^{2} A_{i}\right)^{3}+\frac{1}{24} \tau^{4}\left(\sum_{i=1}^{2} A_{i}\right)^{4}+R_{5}(\tau) \\
& =I-\tau A+\frac{1}{2} \tau^{2} A^{2}-\frac{1}{6} \tau^{3} A^{3}+\frac{1}{24} \tau^{4} A^{4}+R_{5}(\tau) .
\end{aligned}
$$

According to formula (4):

$$
U(\tau, A)=I-\tau A+\frac{1}{2} \tau^{2} A^{2}-\frac{1}{6} \tau^{3} A^{3}+\frac{1}{24} \tau^{4} A^{4}+R_{5}(\tau, A) .
$$

According to condition (b) of the second inequality of the theorem, for $R_{5}(\tau, A)$, the following estimate holds:

$$
\left\|R_{5}(\tau, A) \varphi\right\| \leq c \mathrm{e}^{\omega \tau} \tau^{5}\left\|A^{5} \varphi\right\| \leq c \mathrm{e}^{\omega \tau} \tau^{5}\|\varphi\|_{A^{5}} .
$$

According to equalities (25) and (26):

$$
U(\tau, A)-V(\tau)=R_{5}(\tau, A)-R_{5}(\tau) .
$$

From here, taking into account inequalities (9) and (27), we will obtain the following estimate:

$$
\|[U(\tau, A)-V(\tau)] \varphi\| \leq c \mathrm{e}^{\omega_{2} \tau} \tau^{5}\|\varphi\|_{A^{5}} .
$$

From equalities (2) and (6), taking into account inequalities (7) and (28), we will obtain:

$$
\begin{aligned}
\left\|u\left(t_{k}\right)-u_{k}\left(t_{k}\right)\right\| & =\left\|\left[U\left(t_{k}, A\right)-V^{k}(\tau)\right] \varphi\right\|=\left\|\left[U^{k}(\tau, A)-V^{k}(\tau)\right] \varphi\right\| \\
& =\left\|\left[\sum_{i=1}^{k} V^{k-i}(\tau)[U(\tau, A)-V(\tau)] U((i-1) \tau, A)\right] \varphi\right\| \\
& \leq \sum_{i=1}^{k}\|V(\tau)\|^{k-i}\|[U(\tau, A)-V(\tau)] U((i-1) \tau, A) \varphi\| \\
& \leq \sum_{i=1}^{k} \mathrm{e}^{\omega_{1}(k-i) \tau} c \mathrm{e}^{\omega_{2} \tau} \tau^{5}\|U((i-1) \tau, A) \varphi\|_{A^{5}} \\
& \leq c \mathrm{e}^{\omega_{0} t_{k}} \tau^{5} \sum_{i=1}^{k}\|U((i-1) \tau, A) \varphi\|_{A^{5}} \leq k c \mathrm{e}^{\omega_{0} t_{k}} \tau^{5} \sup _{s \in\left[o, t_{k}\right]}\|U(s, A) \varphi\|_{A^{5}} \\
& \leq c \mathrm{e}^{\omega_{0} t_{k}} t_{k} \tau^{4} \sup _{s \in\left[o, t_{k}\right]}\|U(s, A) \varphi\|_{A^{5}} .
\end{aligned}
$$

Remark 4. In case of a Hilbert space, if $A_{1}, A_{2}$ and $A_{1}+A_{2}$ are self adjoint nonnegative operators, then $\omega_{0}$ will be replaced by 0 in the estimate of the theorem. In addition, for the solution operator of the split problem, the following estimate holds: $\left\|V^{k}(\tau)\right\| \leq 1$.

Remark 5. In case of a Hilbert space, if $A_{1}, A_{2}$ and $A_{1}+A_{2}$ are self adjoint positive defined operators, then $\omega_{0}$ will be replaced by $\left(-\alpha_{0}\right), \alpha_{0}>0$ in the estimate of the Theorem. In addition, for the solution operator of the split problem, the following estimate holds: $\left\|V^{k}(\tau)\right\| \leq \mathrm{e}^{-\alpha_{1} t_{k}}, \alpha_{1}>0$. 
Remark 6. According to the classical theorem of Hille-Philips-Iosida (see [40]), if the operator $(-A)$ generates a strongly continuous semigroup, then the second inequality of condition (b) of the Theorem is automatically satisfied. The proof of this inequality is based on the uniform boundedness principle, due to which constants $M$ and $\omega$ exist, but can not be explicitly constructed (following to method of the proof). For this reason we demand a satisfaction of the second inequality of the condition (b) of the theorem.

\section{ConneCtion Between DeCOMposition formulas With DifFERENT ACCURACIES}

It is interesting if there exists a certain regularity, on the basis of which it is available to construct automatically stable decomposition formulas with accuracy of any order. Concerning the above-mentioned let us consider the concrete first and second order accuracy decomposition formulas and see whether there exists a connection between them.

$$
\begin{aligned}
V^{(1)}(\tau) & =U\left(\tau, A_{1}\right) U\left(\tau, A_{2}\right) \\
V^{(2)}(\tau) & =U\left(\tau, \frac{1}{2} A_{1}\right) U\left(\tau, A_{2}\right) U\left(\tau, \frac{1}{2} A_{1}\right)
\end{aligned}
$$

In this formula and the formulas given below, the upper indices of the operator $V$ denote the order of the corresponding decomposition formula. Formula (29) represents the first order accuracy decomposition formula (see [49]), while formula (30) represents the second order accuracy decomposition formula (see [3]). In order to show more clearly the connection between them, let us rewrite formula (30) in the following form:

$$
\begin{aligned}
V^{(2)}(\tau) & =\left[U\left(\tau, \frac{1}{2} A_{1}\right) U\left(\tau, \frac{1}{2} A_{2}\right)\right]\left[U\left(\tau, \frac{1}{2} A_{2}\right)\left(\tau, \frac{1}{2} A_{1}\right)\right] \\
& =V^{(1)}\left(\frac{1}{2} \tau\right) \overline{V^{(1)}}\left(\frac{1}{2} \tau\right) .
\end{aligned}
$$

In this formula and the formulas given below, we denote by $\bar{V}$ the multiplication of factors of the operator $V$ in the reverse order.

The regularity of the same type exists between the third and fourth order accuracy decomposition formulas, constructed by us (see $[16,18,19]$ ). In order to show this, let us introduce the following notations:

$$
\begin{aligned}
& V^{(3)}(\tau)=\frac{1}{2}\left[V_{1}^{(3)}(\tau)+V_{2}^{(3)}(\tau)\right], \\
& V_{1}^{(3)}(\tau)=U\left(\tau, \alpha A_{1}\right) U\left(\tau, A_{2}\right) U\left(\tau, \bar{\alpha} A_{1}\right), \\
& V_{2}^{(3)}(\tau)=U\left(\tau, \alpha A_{2}\right) U\left(\tau, A_{1}\right) U\left(\tau, \bar{\alpha} A_{2}\right),
\end{aligned}
$$

and

$$
\begin{aligned}
& V^{(4)}(\tau)=\frac{1}{2}\left[V_{1}^{(4)}(\tau)+V_{2}^{(4)}(\tau)\right], \\
& V_{1}^{(4)}(\tau)=U\left(\tau, \frac{\alpha}{2} A_{1}\right) U\left(\tau, \frac{1}{2} A_{2}\right) U\left(\tau, \bar{\alpha} A_{1}\right) U\left(\tau, \frac{1}{2} A_{2}\right) U\left(\tau, \frac{\alpha}{2} A_{1}\right), \\
& V_{2}^{(4)}(\tau)=U\left(\tau, \frac{\alpha}{2} A_{2}\right) U\left(\tau, \frac{1}{2} A_{1}\right) U\left(\tau, \bar{\alpha} A_{2}\right) U\left(\tau, \frac{1}{2} A_{1}\right) U\left(\tau, \frac{\alpha}{2} A_{2}\right) .
\end{aligned}
$$


In order to reveal the connection between formulas (31) and (32), let rewrite the addends of formula (32) in the following form:

$$
\begin{aligned}
V_{1}^{(4)}(\tau) & =\left[U\left(\tau, \frac{\alpha}{2} A_{1}\right) U\left(\tau, \frac{1}{2} A_{2}\right) U\left(\tau, \frac{\bar{\alpha}}{2} A_{1}\right)\right]\left[U\left(\tau, \frac{\bar{\alpha}}{2} A_{1}\right) U\left(\tau, \frac{1}{2} A_{2}\right) U\left(\tau, \frac{\alpha}{2} A_{1}\right)\right] \\
& =V_{1}^{(3)}\left(\frac{1}{2} \tau\right) \overline{V_{1}^{(3)}}\left(\frac{1}{2} \tau\right), \\
V_{2}^{(4)}(\tau) & =\left[U\left(\tau, \frac{\alpha}{2} A_{2}\right) U\left(\tau, \frac{1}{2} A_{1}\right) U\left(\tau, \frac{\bar{\alpha}}{2} A_{2}\right)\right]\left[U\left(\tau, \frac{\bar{\alpha}}{2} A_{2}\right) U\left(\tau, \frac{1}{2} A_{1}\right) U\left(\tau, \frac{\alpha}{2} A_{2}\right)\right] \\
& =V_{2}^{(3)}\left(\frac{1}{2} \tau\right) \overline{V_{2}^{(3)}}\left(\frac{1}{2} \tau\right) .
\end{aligned}
$$

Finally we obtain:

$$
V^{(4)}(\tau)=\frac{1}{2}\left[V_{1}^{(3)}\left(\frac{1}{2} \tau\right) \overline{V_{1}^{(3)}}\left(\frac{1}{2} \tau\right)+V_{2}^{(3)}\left(\frac{1}{2} \tau\right) \overline{V_{2}^{(3)}}\left(\frac{1}{2} \tau\right)\right] .
$$

Unfortunately, the following formula constructed by the same rule:

$$
\begin{aligned}
V^{(5)}(\tau)= & \frac{1}{2}\left[V_{1}^{(4)}\left(\frac{1}{2} \tau\right) \overline{V_{1}^{(4)}}\left(\frac{1}{2} \tau\right)+V_{2}^{(4)}\left(\frac{1}{2} \tau\right) \overline{V_{2}^{(4)}}\left(\frac{1}{2} \tau\right)\right] \\
= & \frac{1}{2}\left[U\left(\tau, \frac{\alpha}{4} A_{1}\right) U\left(\tau, \frac{1}{4} A_{2}\right) U\left(\tau, \frac{\bar{\alpha}}{2} A_{1}\right) U\left(\tau, \frac{1}{4} A_{2}\right) U\left(\tau, \frac{\alpha}{2} A_{1}\right)\right. \\
& \times \\
& U\left(\tau, \frac{1}{4} A_{2}\right) U\left(\tau, \frac{\bar{\alpha}}{2} A_{1}\right) U\left(\tau, \frac{1}{4} A_{2}\right) U\left(\tau, \frac{\alpha}{4} A_{1}\right) \\
& +U\left(\tau, \frac{\alpha}{4} A_{2}\right) U\left(\tau, \frac{1}{4} A_{1}\right) U\left(\tau, \frac{\bar{\alpha}}{2} A_{2}\right) U\left(\tau, \frac{1}{4} A_{1}\right) U\left(\tau, \frac{\alpha}{2} A_{2}\right) \\
& \left.\times U\left(\tau, \frac{1}{4} A_{1}\right) U\left(\tau, \frac{\bar{\alpha}}{2} A_{2}\right) U\left(\tau, \frac{1}{4} A_{1}\right) U\left(\tau, \frac{\alpha}{4} A_{2}\right)\right] .
\end{aligned}
$$

does not represent the fifth order accuracy decomposition formula. To check this out, it is sufficient to calculate, for example, the coefficient $\left[V^{(5)}(\tau)\right]_{1,2,1,2,1}$. We see that

$$
\left[V^{(5)}(\tau)\right]_{1,2,1,2,1} \neq \frac{1}{5 !}
$$

In our opinion, it is interesting and important to find the general regularity, by means of which it will be available to construct recurrently an automatically stable decomposition formula with accuracy of any order, or to prove that, on the complex number field, there does not exist an automatically stable decomposition formula with accuracy of order more than four (as well as on the real number field there does not exist an automatically stable decomposition formula with accuracy of order more than two). In addition, it is not excluded that, to obtain the higher order accuracy, it will be necessary to use as split parameters, for example, quaternions instead of complex numbers,

In our opinion, these questions are very interesting and difficult, and we work in this direction, but we have not yet obtain actual results.

\section{Conclusion}

In case when operators $A_{1}, A_{2}$ are matrices, it is obvious that conditions of the theorem are automatically satisfied. Also conditions of the Theorem are satisfied if $A_{1}, A_{2}$ and $A$ are self-adjoint, positive definite operators. 
The requirement, that the operators $\alpha A$ and $\bar{\alpha} A\left(\alpha=1 / \sqrt{3}\left(\cos 30^{\circ}+i \sin 30^{\circ}\right)\right)$ must generate a strongly continuous semigroup, puts the condition on the spectrum of $A$. Namely, the spectrum of $A$ must be placed within a sector with the angle less than 120 degrees, because in case of turning of spectrum by \pm 30 degrees (this is caused by multiplying of $A$ on the parameters $\alpha$ and $\bar{\alpha}$ ) the spectrum area will stay in the positive (right) half-plane.

The fourth order accuracy is reached by introducing a complex parameter. For this reason, each equation of the given decomposed system is replaced by a pair of real equations, unlike the lower order accuracy schemes. To solve the concrete problem, (for example) the matrix factorization can be used, where coefficients are the matrices of the second order, while in the lower order accuracy schemes the common factorization may be used.

It must be noted that a sum of the absolute values of coefficients of the addends of the transition operator $V(\tau)$ equals to one, unlike the high order accuracy decomposition schemes considered in [8]. Hence, the considered scheme is stable for any bounded operators $A_{1}, A_{2}$.

\section{REFERENCES}

[1] V.B. Andreev, On difference schemes with a splitting operator for general $p$-dimensional parabolic equations of second order with mixed derivatives. SSSR Comput. Math. Math. Phys. 7 (1967) 312-321.

[2] G.A. Baker, An implicit, numerical method for solving the two-dimensional heat equation. Quart. Appl. Math. 17 (1959/1960) 440-443.

[3] G.A. Baker and T.A. Oliphant, An implicit, numerical method for solving the two-dimensional heat equation. Quart. Appl. Math. 17 (1959/1960) 361-373.

[4] G. Birkhoff and R.S. Varga, Implicit alternating direction methods. Trans. Amer. Math. Soc. 92 (1959) 13-24.

[5] G. Birkhoff, R.S. Varga and D. Young, Alternating direction implicit methods. Adv. Comput. Academic Press, New York 3 (1962) 189-273.

[6] P.R. Chernoff, Note on product formulas for operators semigroups. J. Functional Anal. 2 (1968) 238-242.

[7] P.R. Chernoff, Semigroup product formulas and addition of unbounded operators. Bull. Amer. Mat. Soc. 76 (1970) $395-398$.

[8] B.O. Dia and M. Schatzman, Comutateurs semi-groupes holomorphes et applications aux directions alternées. RAIRO Modél. Math. Anal. Numér. 30 (1996) 343-383.

[9] E.G. Diakonov, Difference schemes with a splitting operator for nonstationary equations. Dokl. Akad. Nauk SSSR 144 (1962) $29-32$.

[10] E.G. Diakonov, Difference schemes with splitting operator for higher-dimensional non-stationary problems. SSSR Comput. Math. Math. Phys. 2 (1962) 549-568.

[11] J. Douglas, On numerical integration of by impilicit methods. SIAM 9 (1955) 42-65.

[12] J. Douglas and H. Rachford, On the numerical solution of heat condition problems in two and three space variables. Trans. Amer. Math. Soc. 82 (1956) 421-439.

[13] M. Dryja, Stability in $W_{2}^{2}$ of schemes with splitting operators. SSSR. Comput. Math. Math. Phys. 7 (1967) $296-302$.

[14] G. Fairweather, A.R. Gourlay and A.R. Mitchell, Some high accuracy difference schemes with a splitting operator for equations of parabolic and elliptic type. Numer. Math. 10 (1967) 56-66.

[15] I.V. Fryazinov, Increased precision order economical schemes for the solution of parabolic type multi-dimensional equations. SSSR. Comput. Math. Math. Phys. 9 (1969) 1319-1326.

[16] Z.G. Gegechkori, J.L. Rogava and M.A. Tsiklauri, High-degree precision decomposition method for an evolution problem. Tbilisi, Reports of Enlarged Session of the Seminar of I. Vekua Institute of Applied Mathematics 14 (1999) 45-48.

[17] Z.G. Gegechkori, J.L. Rogava and M.A. Tsiklauri, High degree precision decomposition formulas of semigroup approximation. Tbilisi, Reports of Enlarged Session of the Seminar of I. Vekua Institute of Applied Mathematics 16 (2001) 89-92.

[18] Z.G. Gegechkori, J.L. Rogava and M.A. Tsiklauri, Sequention-Parallel method of high degree precision for Cauchy abstract problem solution. Minsk, Comput. Methods in Appl. Math. 1 (2001) 173-187.

[19] Z.G. Gegechkori, J.L. Rogava and M.A. Tsiklauri, High degree precision decomposition method for the evolution problem with an operator under a split form. ESAIM: M2AN 36 (2002) 693-704.

[20] D.G. Gordeziani, On application of local one-dimensional method for solving parabolic type multi-dimensional problems of 2m-degree, Proc. of Science Academy of GSSR 3 (1965) 535-542.

[21] D.G. Gordeziani and A.A. Samarskii, Some problems of plates and shells thermo elasticity and method of summary approximation. Complex analysis and it's applications (1978) 173-186.

[22] D.G. Gordeziani and H.V. Meladze, On modeling multi-dimensional quasi-linear equation of parabolic type by one-dimensional ones, Proc. of Science Academy of GSSR 60 (1970) 537-540.

[23] D.G. Gordeziani and H.V. Meladze, On modeling of third boundary value problem for the multi-dimensional parabolic equations of arbitrary area by the one-dimensional equations. SSSR Comput. Math. Math. Phys. 14 (1974) 246-250. 
[24] A.R. Gourlay and A.R. Mitchell, Intermediate boundary corrections for split operator methods in three dimensions. Nordisk Tidskr. Informations-Behandling 7 (1967) 31-38.

[25] N.N. Ianenko, On Economic Implicit Schemes (Fractional steps method). Dokl. Akad. Nauk SSSR 134 (1960) 84-86.

[26] N.N. Ianenko, Fractional steps method of solving for multi-dimensional problems of mathematical physics. Novosibirsk, Nauka (1967).

[27] N.N. Ianenko and G.V. Demidov, The method of weak approximation as a constructive method for building up a solution of the Cauchy problem. Izdat. "Nauka", Sibirsk. Otdel., Novosibirsk. Certain Problems Numer. Appl. Math. (1966) 60-83.

[28] T. Ichinose and S. Takanobu, The norm estimate of the difference between the Kac operator and the Schrodinger emigroup. Nagoya Math. J. 149 (1998) 53-81.

[29] T. Ichinose and H. Tamura, The norm convergence of the Trotter-Kato product formula with error bound. Commun. Math. Phys. 217 (2001) 489-502.

[30] V.P. Ilin, On the splitting of difference parabolic and elliptic equations. Sibirsk. Mat. Zh 6 (1965) 1425-1428.

[31] K. Iosida, Functional analysis. Springer-Verlag (1965).

[32] T. Kato, The theory of perturbations of linear operators. Mir (1972).

[33] A.N. Konovalov, The fractional step method for solving the Cauchy problem for an $n$-dimensional oscillation equation. Dokl. Akad. Nauk SSSR 147 (1962) 25-27.

[34] S.G. Krein, Linear equations in Banach space. Nauka (1971).

[35] A.M. Kuzyk and V.L. Makarov, Estimation of an exactitude of summarized approximation of a solution of Cauchy abstract problem. Dokl. Akad. Nauk USSR 275 (1984) 297-301.

[36] G.I. Marchuk, Split methods. Nauka (1988).

[37] G.I. Marchuk and N.N. Ianenko, The solution of a multi-dimensional kinetic equation by the splitting method. Dokl. Akad. Nauk SSSR 157 (1964) 1291-1292.

[38] G.I. Marchuk and U.M. Sultangazin, On a proof of the splitting method for the equation of radiation transfer. SSSR. Comput. Math. Math. Phys. 5 (1965) 852-863.

[39] D. Peaceman and H. Rachford, The numerical solution of parabolic and elliptic differential equations. SIAM 3 (1955) $28-41$.

[40] M. Reed and B. Simon, Methods of modern mathematical physics. II. Fourier analysis, self-adjointness. New York-London, Academic Press [Harcourt Brace Jovanovich, Publishers] (1975).

[41] J.L. Rogava, On the error estimation of Trotter type formulas in the case of self-Andjoint operator. Functional analysis and its aplication 27 (1993) 84-86.

[42] J.L. Rogava, Semi-discrete schemes for operator differential equations. Tbilisi, Georgian Technical University press (1995).

[43] A.A. Samarskii, On an economical difference method for the solution of a multi-dimensional parabolic equation in an arbitrary region. SSSR Comput. Math. Math. Phys. 2 (1962) 787-811.

[44] A.A. Samarskii, On the convergence of the method of fractional steps for the heat equation. SSSR Comput. Math. Math. Phys. 2 (1962) 1117-1121.

[45] A.A. Samarskii, Locally homogeneous difference schemes for higher-dimensional equations of hyperbolic type in an arbitrary region. SSSR Comput. Math. Math. Phys. 4 (1962) 638-648.

[46] A.A. Samarskii, P.N. Vabishchevich, Additive schemes for mathematical physics problems. Nauka (1999).

[47] Q. Sheng, Solving linear partial differential equation by exponential spliting. IMA J. Numerical Anal. 9 (1989) 199-212.

[48] R. Temam, Sur la stabilité et la convergence de la méthode des pas fractionnaires. Ann. Mat. Pura Appl. 4 (1968) $191-379$.

[49] H. Trotter, On the product of semigroup of operators. Proc. Amer. Mat. Soc. 10 (1959) 545-551.

To access this journal online:

www.edpsciences.org 